\title{
The Evolution of the Gas Content of Galaxy Groups
}

\section{Eric M. Wilcots*}

University of Wisconsin-Madison

E-mail: ewilcotseastro.wisc.edu

\section{Emily Freeland \& Kelley Hess}

University of Wisconsin-Madison

E-mail: freelandeastro.wisc.edu, hesseastro.wisc.edu

\begin{abstract}
The current state of our understanding of the nature of the baryon content of galaxy groups, derived largely from a large body of X-ray observations, leaves us with two key questions. First, what are the relative fractions of the hot, warm/hot, and neutral gas in galaxy groups, and how is each phase distributed within groups? Second, how has the baryon content of galaxy groups evolved over time and what is its relationship to the dynamical evolution of the group? We present the results from a number of investigations of the HI content, the properties of bent-double radio sources in groups, and deep searches for diffuse synchrotron emission in groups are now shedding light on the evolution of the baryon content of galaxy groups.
\end{abstract}

From planets to dark energy: the modern radio universe October 1-5 2007

University of Manchester, Manchester, UK

\footnotetext{
* Speaker.
} 


\section{Introduction - Why Galaxy Groups}

Nearly $70 \%$ of galaxies reside in groups [13] and, therefore, understanding the evolution of galaxy groups is crucial to understanding the evolution of galaxies in general. The goal of the work described here is to probe both how the evolution of the group affects the evolution of the resident galaxies and how the evolution of the galaxies affects the group. The definition of a "group", however, is not straightforward. A number of different authors have applied a range of criteria, few of which are particularly robust. For the purposes of this presentation we will adopt the definition of group as having a dark matter halo mass of $10^{12}-10^{14} \mathrm{M}_{\odot}$ [5]. For dynamical systems with a radius of $\sim 0.5 \mathrm{Mpc}$, this translates into a velocity dispersion of a few hundred $\mathrm{km} \mathrm{s}^{-1}$.

Regardless of the formal definition, groups are not simply scaled down versions of clusters. Because the velocity dispersions of groups are of the same order as the internal velocities of the individual galaxies, mergers between galaxies are much more likely in the group environment, while processes like "harrassment" are much less likely to play a role in the evolution of the gas content of groups. We discuss three aspects of the evolution of the gas content of galaxy groups: the relationship between the distribution of the neutral gas and the dynamical state of the group; the density of the intergalactic medium in groups; and the heating of the intragroup medium by AGN.

\section{What is the Evolution of the HI Content of Galaxy Groups?}

To investigate the evolution of the H I content of galaxy groups we initiated a survey of 10 groups spanning a range of morphological properties, ranging from groups with a spiral fraction of $100 \%$ to those with spiral fractions of less than $20 \%[8,14]$. The selection reflects the observation that groups dominated by elliptical galaxies are also those that typically harbor X-ray halos; in fact, no group with a spiral fraction of $100 \%$ has even been seen to have an X-ray halo [11].

In groups with a significant X-ray halo (e.g. NGC 2563) we see that H I rich galaxies only reside outside of the extent of the X-ray halo [14]. Groups with no elliptical galaxies and no detectable X-ray halos have $\mathrm{H}$ I properties that are indistinguishable from those of galaxies in the field. It is the groups with a mixed population of ellipticals and spirals in which we find evidence of the ongoing evolution of neutral gas content. One example of this is in GH89 where almost all of the $\mathrm{H}$ I detected galaxies are either currently participating in strong interactions or have significantly distorted $\mathrm{H}$ I distributions that are suggestive of recent interactions as in Figure 1 and [8]. We can conclude that one ramification of the dynamical evolution of galaxy groups is the deposition of a significant amount of neutral gas into the intergalactic medium.

Our results are also consistent with the general picture that the gas content of spiral-rich groups is largely in the form of neutral $\mathrm{H}$ I while the gas content of elliptical-dominated groups is typically hot, X-ray emitting gas. Transformation of the nature of the gas content of the groups goes along with a dynamical evolution that transforms spiral-rich groups into elliptical-dominated ones. An interesting point, however, is that we find the gas mass of X-ray luminous groups [9] to be $\sim 10$ times larger than the neutral gas mass of spiral-rich groups of comparable dynamical mass. If, in fact, groups evolve dynamically from being spiral-dominated to being elliptical-dominated, this result suggests that spiral rich groups in particular may harbor a heretofore unseen gas component of significant mass that is heated to X-ray emitting temperatures by the processes that drive the 
overall dynamical evolution of the group. Resolving this issue requires a better understanding of the total baryon content of groups.

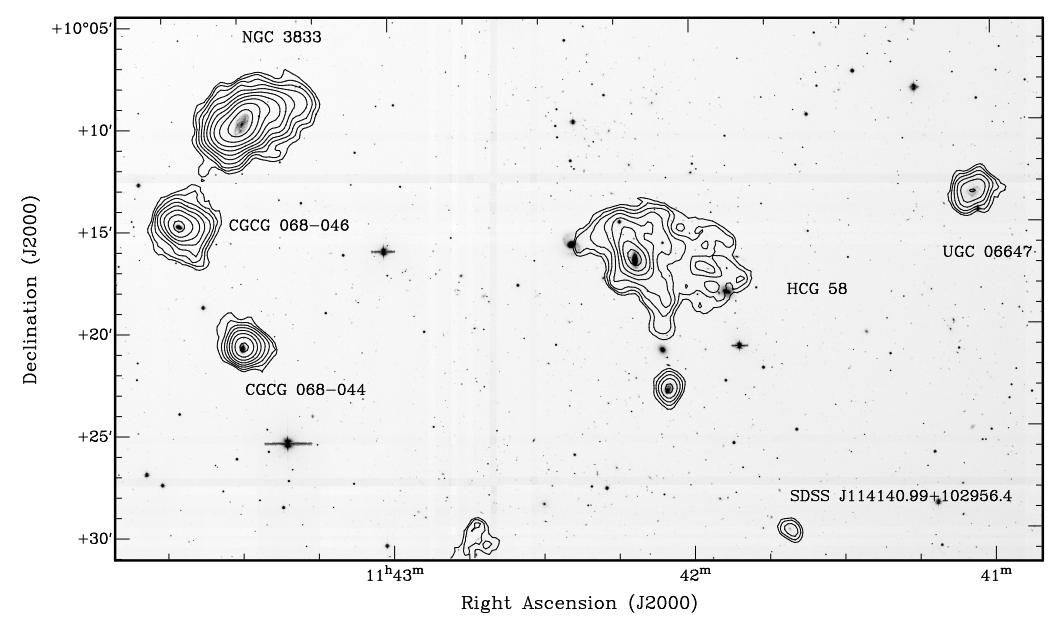

Figure 1: Figure 2. The H I content of the GH89 group. This is a group with mixed morphology, but no detectable X-ray halo. As is common for groups of this type, most of the galaxies are involved in gravitational interactions.

\section{Measuring the Density of the Intergalactic Medium in Galaxy Groups}

While the results of H I surveys of groups may suggest that some groups contain a significant amount of baryonic matter that is not readily observable, simulations of the formation of galaxies and large scale structure also predict the existence of a "warm-hot intergalactic medium" (WHIM) that contains a large fraction of the baryons in the Universe [2]. Direct detection of this gas, however, has been difficult. The approach we take is to use the properties of bent-tail radio sources as probes of the density of the intergalactic medium. Bent, or wide-angle tail, sources are believed to be the result of ram pressure on the jets of radio galaxies, either from the motion of the radio galaxy through the intracluster medium (ICM), or, from the flow of the ICM around the radio galaxy [1].

Freeland \& Wilcots [7] initiated a large survey of bent-doubles using a combination of data from the FIRST survey, new radio continuum observations using the GMRT, and optical spectroscopy obtained with the WIYN ${ }^{1} 3.5 \mathrm{~m}$ telescope. The ultimate goal is to use the curvature of the jets and the velocity dispersion of the group to measure the density of the intragroup medium. With an initial comparison with photometric redshifts, we ruled out all bent-doubles residing in known clusters or high density environments. The complementary radio continuum and optical observations were restricted to those bent-doubles residing in low density environments. An example of one of our sources is shown in Figure 2.

\footnotetext{
${ }^{1}$ The WIYN Observatory is a joint facility of the University of Wisconsin-Madison, Indiana University, Yale University, and the National Optical Astronomy Observatories.
} 


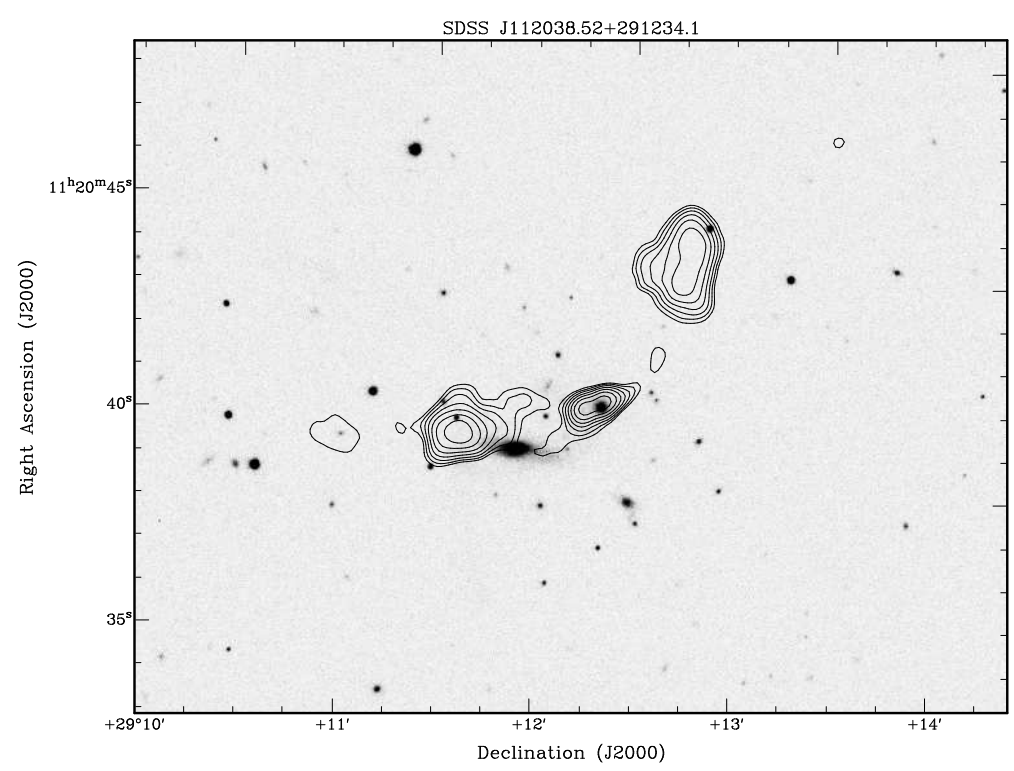

Figure 2: The $610 \mathrm{MHz}$ radio continuum contours are overlayed on a SDSS image of one of the bent-doubles in the sample

For our initial three bent-doubles we find mean IGM densities of $9.3 \times 10^{-05}-1.0 \times 10^{-04}$ $\mathrm{cm}^{-3}$ [6]. These densities are consistent with earlier results on two "poor clusters" [6] and with predictions for the WHIM [4] and suggest that the IGM in galaxy groups, even groups with no diffuse X-ray emission, contains a significant fraction of the baryonic content of the Universe.

\section{The Role of AGN in Heating the Intragroup Medium}

The final component of our investigation of the evolution of the gas content of galaxy groups is to understand the nature of the excess heating required to account for the observed X-ray properties of the intragroup medium. From a wealth of X-ray observations of galaxy groups it is apparent that the relationship between the X-ray luminosity and the X-ray temperature is steeper than what one would expect from pure gravitational infall . The excess entropy is believed to primarily arise from heating by AGN, though the evidence is not yet conclusive [3].

One approach [15] is to measure what fraction of local galaxy groups contained an AGN by comparing group catalogs [5] with the Sadler et al. [12] catalog of radio sources found in the $2 \mathrm{dF}$ Galaxy Redshift Survey. Limiting ourselves to a redshift range of $0.03 \leq z \leq 0.3$, we found that $70 \%$ of the AGN reside in 2dFGRS galaxy groups [15]. $7.7 \%$ of groups with at least 4 members and $4.5 \%$ of all groups contained an AGN, but significantly fewer than $1 \%$ of groups contained more than one AGN. Even with the relatively high flux limited employed [12], it is not obvious that AGN can currently have a tremendous impact on the overall population of galaxy groups.

Given that the statistical approach to estimating the impact of AGN on the intragroup medium is not conclusive, we also initiated a campaign to directly measure AGN feedback via the detection of low frequency radio continuum emission arising from the intragroup medium. 


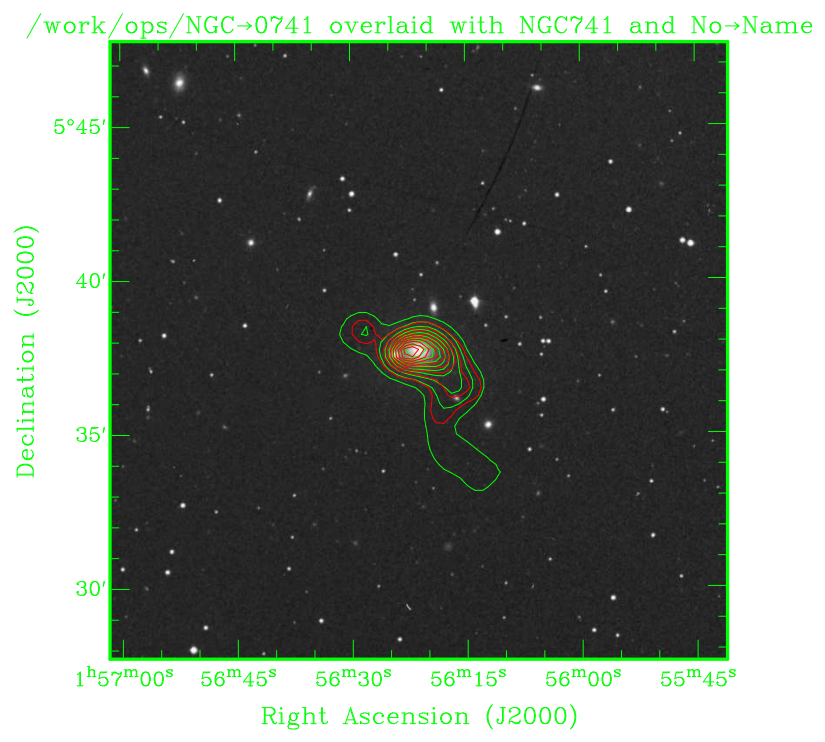

Figure 3: We show the comparison of the $327 \mathrm{MHz}$ and $1420 \mathrm{MHz}$ radio continuum emission associated with the radio galaxy NGC 741. The contours are overlayed on an optical image retrieved from the Digital Sky Survey. The observation that the lower frequency emission is more extended than the $1420 \mathrm{MHz}$ is indicative of aging synchrotron emission and older AGN activity.

Wilcots, Hess, \& Grcevich [15] reported on the initial results of a study of low frequency emission in X-ray luminous galaxy groups harboring radio galaxies. In Figure 3 we show a comparison of our VLA $327 \mathrm{MHz}$ (green) data for the NGC 741 group with the $1420 \mathrm{MHz}$ image from the NVSS (red). The comparison between the emission at the two different frequencies shows that the lower frequency emission is more extended than that at $1420 \mathrm{MHz}$. In addition, the spectral index, $\alpha$ where the intensity goes as $S \propto v^{\alpha}$, steepens from -0.51 near the center of the source to -0.90 near the outer edge of the emission. This is what one would expect given an aging synchrotron population; the more extended low frequency emission traces an earlier epoch of AGN activity. Deeper low frequency observations should yield accurate estimates of the past AGN activity and a measure of how much energy jets have contributed to the intragroup medium.

\section{Conclusions}

From the multi-faceted approach presented here we see that the gas content of galaxy groups is closely linked to the overall dynamical state of the group. Dynamically young systems, those without diffuse X-ray emission or a significant fraction of elliptical galaxies, have most their observable gas in the form of H I. Groups with a modest elliptical fraction $(\sim 0.5)$ are most likely to have ongoing mergers and, thus, copious amounts in intergalactic H I. In the most dynamically evolved systems we find that the $\mathrm{H}$ I detected galaxies tend to reside outside of the X-ray emitting 
halo. A comparison of groups with similar dynamical mass shows that X-ray rich groups have a higher total gas content than groups whose observable gas content is entirely neutral.

It does appear that galaxy groups contain a significant amount of gas in the intragroup medium. The densities of the intragroup medium derived from considering the bending angle of bent-jets and the velocity dispersion of the groups imply that groups contain a large reservoir of undetected baryons in their intergalactic medium. If this is a common property of all groups this gas represents a significant fraction of the total baryon content of the local Universe. Whether this gas is the same gas that is heated to observable temperatures in X-ray luminous groups has yet to be determined.

While there is significant evidence that AGN are responsible for heating the intragroup medium, radio observations of X-ray luminous groups yield mixed results. There is extended low frequency radio continuum emission arising from the intergalactic medium in groups with active radio galaxies. The extent to which this emission is responsible for the elevated temperatures and entropy in such groups remains to be seen.

\section{References}

[1] Burns, J.O., Loken, C., Roettiger, K., Rizza, E., Bryan, G., Norman, M.L., Gomez, P., \& Owen, F.N. 2002, Stormy Weather and Cluster Radio Galaxies, NewAR 46 (135)

[2] Cen, R., Tripp, T.M., Ostriker, J.P., \& Jenkins, E.B. 2001, Revealing the Warm-Hot Intergalactic Medium with $O$ VI Absorption, ApJ 559 (L5)

[3] Croston, J.H., Hardcastle, M.J., \& Birkinshaw, M., 2005, Evidence for Radio-Source Heating of Groups, MNRAS 357 (279)

[4] Dave',R., Cen, R., Ostriker, J.P., Bryan, G.L., Hernquist, L., Katz, N., Weinberg, D.H., Norman, M.L., \& O'Shea, B. 2001, Baryons in the Warm-Hot Intergalactic Medium, ApJ 552 (473)

[5] Eke, V.R. et al., 2004, Galaxy Groups in the Two-degree Field Galaxy Redshift Survey: The Luminous Content of the Groups, MNRAS, 355 (769)

[6] Ekers, R.D. 1978, Two Head-Tail Radio Sources in Poor Clusters, A\&A 69 (253)

[7] Freeland, E.E., \& Wilcots, E.M., 2007, Bent-Double Radio Sources as Probes of the Intragroup Medium in Groups of Galaxies, BAAS 211 (9611).

[8] Freeland, E.E., Wilcots, E.M., \& Stilp, A.M. 2008, in preparation.

[9] Helsdon, S.F., Ponman, T.J., \& Mulchaey, J.S. 2005, Chandra Observations of Low Velocity

Dispersion Groups, ApJ 618 (679)

[10] Jeltema, T.E., Mulchaey, J.S., Lubin, L.M., Rosati, P., \& Bohringer, H. 2006, ApJ 649 (649)

[11] Mulchaey, J.S., Davis, D.S., Mushotzky, R.F., \& Burstein, D. 2003, An X-ray Atlas of Groups of Galaxies, ApJS 145 (39).

[12] Sadler, E.M. et al. 2002, Radio Sources in the 2dF Galaxy Redshift Survey - II. Local Radio luminosity Functions for AGN and Star-Forming Galaxies at $1.4 \mathrm{GHz}$, MNRAS, 329 (227)

[13] Tully, R.B. 1987, Nearby Groups of Galaxies. II - An All-Sky Survey within 3000 kilometers per second, ApJ 321 (280).

[14] Wilcots, E.M., van Gorkom, J., Mulchaey, J.S., \& Zabludoff, A. 2008, in preparation. [15] Wilcots, E.M., Hess, K.M., \& Grcevich, J. 2007, AGN Heating of the Intergalactic Medium in Galaxy Groups, in "Extragalactic Jets: Theory and Observation from Radio to Gamma Ray, eds T.A Rector and D.S. De Young. 